\title{
Cost-effectiveness analysis of cemiplimab vs pembrolizumab for treatment of advanced cutaneous squamous cell carcinoma
}

\author{
Eleanor Paul, MSc; Gerasimos Konidaris, MSc; Shannon Cope, MSc; Chieh-I Chen, MPH; Sam Keeping, MSc; \\ Yingxin Xu, PhD; Kokuvi Atsou, PhD; Dieter Ayers, MSc; Patricia Guyot, PhD; Medha Sasane, PhD; \\ Ali Mojebi, MD, MHSc; and Andreas Kuznik, PhD
}

\section{What is already known about this subject}

- Before the availability of immunotherapy, patients with advanced cutaneous squamous cell carcinoma (CSCC), which includes metastatic CSCC or locally advanced CSCC not amenable to curative surgery or curative radiation, often had a poor prognosis.

- In 2018, the FDA approved cemiplimab-rwlc as the first programmed cell death-1 monoclonal antibody for the treatment of patients with advanced CSCC.

- We previously reported that from a US perspective, cemiplimab is a cost-effective treatment option vs historical standard of care for advanced CSCC.

\section{What this study adds}

- This study assessed the costeffectiveness of cemiplimab-rwlc compared with pembrolizumab (following its approval by the FDA in June 2020) for recurrent or metastatic CSCC that is not curable by surgery or radiation for the treatment of patients with advanced CSCC in the United States.

- The results of this analysis show that compared with pembrolizumab, cemiplimab offers a cost-effective treatment for patients with advanced CSCC and is expected to provide significant clinical and economic value to health care payers in the United States.

\section{ABSTRACT}

BACKGROUND: Most cutaneous squamous cell carcinomas (CSCCS) can be treated with surgical excision or radiation; however, approximately $1 \%$ of patients develop advanced disease. In 2018, the FDA approved cemiplimab-rwlc as the first programmed cell death-1 (PD-1) monoclonal antibody for the treatment of patients with metastatic CSCC or locally advanced CSCC who are not candidates for curative surgery or curative radiation. In June 2020, pembrolizumab, another PD-1 monoclonal antibody, was approved for the treatment of patients with recurrent or metastatic CSCC who are not candidates for curative surgery or radiation. We previously reported on the cost-effectiveness of cemiplimab vs historical standard of care for the treatment of advanced CSCC from a US perspective.

OBJECTIVE: To estimate the cost-effectiveness of cemiplimab vs pembrolizumab for patients with advanced CSCC in the United States.

\section{Author affiliations \\ Eleanor Paul, MSc; Shannon Cope, MSc; Sam Keeping, MSc; Dieter Ayers, MSc; and Ali Mojebi, MD, MHSc, PRECISIONheor, Vancouver, British Columbia, Canada. Gerasimos Konidaris, MSc, Sanofi, Reading, United Kingdom. Chieh-I Chen, MPH; Yingxin Xu, PhD; and Andreas Kuznik, PhD, Regeneron Pharmaceuticals, Inc., Tarrytown, NY. Kokuvi Atsou, PhD, and Patricia Guyot, $\mathrm{PhD}$, Sanofi, Chilly-Mazarin, France. Medha Sasane, PhD, Sanofi, Bridgewater, NJ.}

AUTHOR CORRESPONDENCE: Sam Keeping, 604.336.3050 ext 67122; Sam.Keeping@precisionvh.com

J Manag Care Spec Pharm 2021;27(11):1513-25

Copyright $\odot 2021$, Academy of Managed Care Pharmacy. All rights reserved.

METHODS: A "partitioned survival" framework was used to assess the cost-effectiveness of cemiplimab vs pembrolizumab. Clinical inputs were based on the most recent data cut of the phase 2 trials for cemiplimab (EMPOWER-CSCC-1; NCT02760498) and pembrolizumab (KEYNOTE-629). Progression-free survival and overall survival were extrapolated using parametric models until all patients had progressed or died. Health state utilities were derived from data collected in the EMPOWER-CSCC-1 trial. Costs included drug acquisition, drug administration, disease 
management, terminal care, and adverse events and were based on published 2020 US list prices. To assess model uncertainty, 1-way sensitivity and probabilistic sensitivity analyses (PSA) were conducted, alongside scenario analyses evaluating key modeling assumptions.

RESULTS: In the base case, cemiplimab resulted in an incremental gain of 3.44 life-years (discounted) and incremental cost-effectiveness ratio (ICER) of $\$ 130,329$ per quality-adjusted life-year (QALY) vs pembrolizumab. At a willingness-to-pay threshold of \$150,000/QALY, PSA indicated a $71 \%$ probability that cemiplimab is cost-effective when compared with pembrolizumab. Scenario analysis resulted in ICERs ranging from $\$ 115,909$ to $\$ 187,374$.

CONCLUSIONS: Findings suggest that cemiplimab is a cost-effective treatment for patients with advanced CSCC, compared with pembrolizumab. These results should be interpreted cautiously in the absence of head-to-head trials; however, in the absence of such data, these results can be used to inform health care decisions over resource allocation.

Cutaneous squamous cell carcinoma (CSCC) is the second most common cancer in the United States, with an estimated incidence of approximately 1.5 million cases per year. ${ }^{1}$ The incidence of CSCC is increasing by approximately $3 \%$ to $7 \%$ per year, ${ }^{2}$ related to increased exposure to ultraviolet (UV) light, which damages the skin. Other known risk factors for CSCC include advanced age, immunosuppression, history of actinic keratosis, and UV-sensitive skin. ${ }^{3-9}$ Although most patients with CSCC can be treated with surgical excision or radiation, approximately $1 \%$ of patients develop advanced disease, which often has a poor prognosis. ${ }^{9-11}$ Before the availability of immunotherapies, median survival for patients with advanced CSCC was limited (ranging between 8.1 and 17.5 months) $)^{12-21}$ with only $16 \%$ to $29 \%$ of patients surviving beyond 3 years. . $^{14-16,18,20}$

In 2018, the US Food and Drug Administration (FDA) approved cemiplimab (as cemiplimab-rwlc) as the first programmed cell death-1 (PD-1) monoclonal antibody for the treatment of patients with metastatic CSCC (mCSCC) or locally advanced CSCC (laCSCC) who are not candidates for curative surgery or curative radiation, collectively known as advanced CSCC. The introduction of cemiplimab to the oncology landscape significantly changed the treatment paradigm for these patients in the United States. ${ }^{22}$ Patients with advanced CSCC can receive treatment with cemiplimab until progression or treatment discontinuation for other reasons. We have previously reported the comparative efficacy and cost-effectiveness of cemiplimab ${ }^{23,24}$ based on clinical trial data for cemiplimab (EMPOWERCSCC-1 [NCT02760498], median follow-up 11.9 months) vs historical standard of care (SOC). In the analysis, the effectiveness of SOC was based on pooled single-arm clinical trials and retrospective studies evaluating chemotherapy and epidermal growth factor receptor (EGFR) inhibitors (cetuximab, erlotinib, and gefitinib). The cost-effectiveness results suggested that from a US perspective, cemiplimab was a cost-effective treatment option vs historical SOC for advanced CSCC. ${ }^{24}$

More recently in June 2020, pembrolizumab, another PD-1 monoclonal antibody, was approved by the FDA for the treatment of patients with recurrent or metastatic CSCC that is not curable by surgery or radiation until disease progression, unacceptable toxicity, or up to 24 months. ${ }^{25}$ Given limited resources in health care in the United States, it is important to evaluate not only the comparative efficacy but also the long-term value provided by recently licensed immunotherapies in the advanced CSCC treatment paradigm. Thus, the objective of this analysis was to estimate the cost-effectiveness of cemiplimab compared with pembrolizumab for the treatment of patients with advanced CSCC in the United States.

\section{Methods}

\section{MODEL STRUCTURE}

The cost-effectiveness model used a "partitioned survival" framework, which is described in more detail in Supplementary Appendix A (available in online article). This model structure is consistent with the cost-effectiveness model previously published by Konidaris et al ${ }^{24}$ and also reflects the most commonly used model structure in health technology assessment evaluations for advanced or metastatic oncologic therapies. ${ }^{26,27}$ The model assumed a lifetime horizon (ie, 30 years). The model used a 1-month cycle length (ie, 30.4 days/month; 365 days/12 months), and a half-cycle correction was included. A discount rate of $3 \%$ was applied to both health outcomes (life-years [LYs] and quality-adjusted LYs [QALYs]) and costs. ${ }^{28}$ The model was programmed in Microsoft Excel version 2013 (Microsoft Corporation).

\section{MODEL INPUTS}

Clinical Evidence. The clinical efficacy of cemiplimab was based on individual patient level data (IPD) from the most recent data cut of the phase 2 cemiplimab trial, EMPOWER-CSCC-1 $(\mathrm{N}=193$, median follow-up 15.7 months), presented at American Society of Clinical Oncology annual meeting 2020. ${ }^{29}$

A systematic literature review was conducted in May 2019 to identify available studies reporting outcomes 


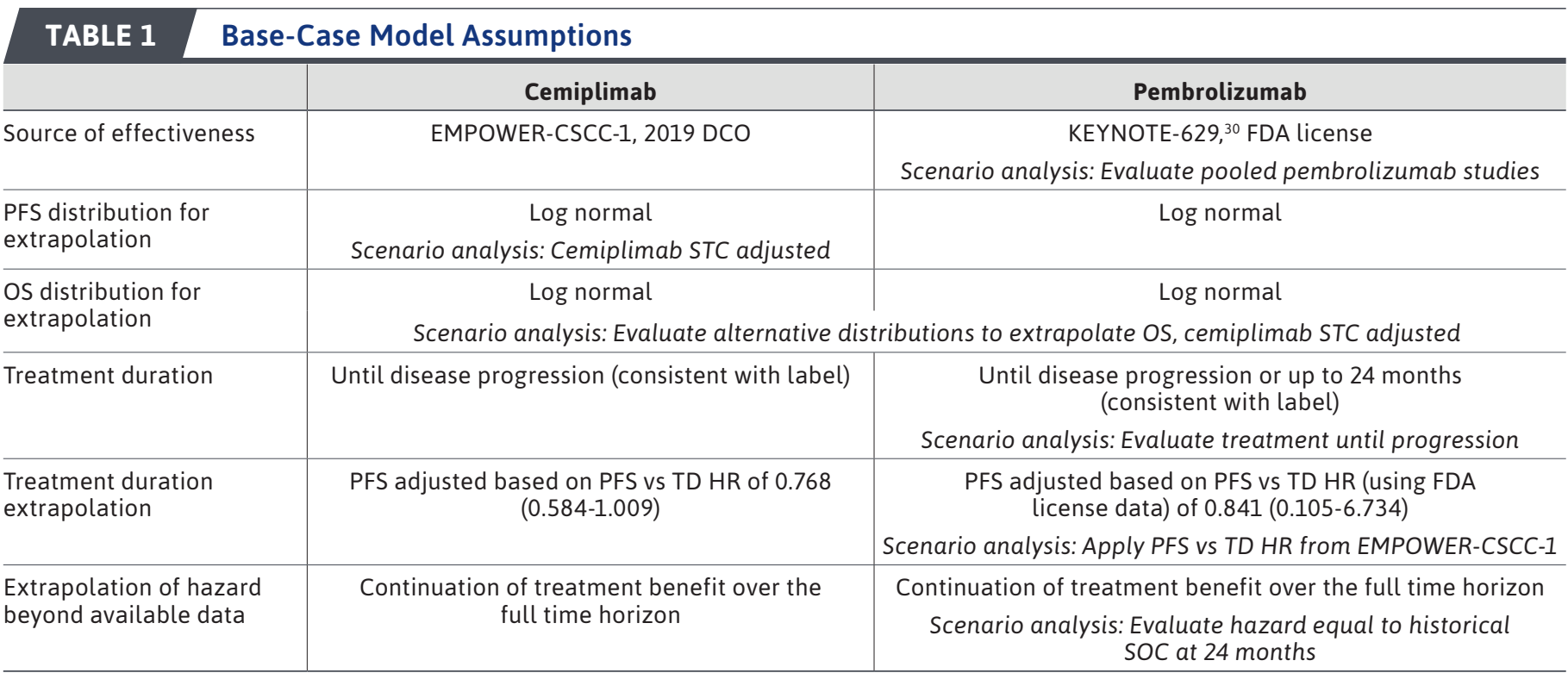

$D C O=$ data cutoff; $F D A=U S$ Food and Drug Administration; $H R=$ hazard ratio; $O S=$ overall survival; $P F S=$ progression-free survival; $S O C=$ standard of care; $S T C=$ simulated treatment comparison; $T D=$ treatment duration.

of systemic therapy for advanced CSCC to inform the comparative clinical efficacy inputs to the model. Study eligibility criteria were defined in terms of the population, interventions, comparisons, outcomes, and study design structure (see Supplementary Appendix B, available in online article). Methods and findings of the review are outlined in Keeping et al. ${ }^{23}$ Additional manual searches were conducted to ensure any reports on the included trials published since the original search were also captured. For pembrolizumab, two phase 2 studies were identified: Grob et al (KEYNOTE-629, $\mathrm{N}=105),{ }^{30}$ the registrational trial of pembrolizumab, and Maubec et al (CARSKIN, $N=39) .{ }^{31}$ The Guyot algorithm was used to reconstruct the progressionfree survival (PFS) and overall survival (OS) IPD from the Kaplan-Meier (KM) curves for each of these trials. ${ }^{32}$

Supplementary Appendix C, Figure C1 (available in online article) illustrates the reported OS and PFS KM curves, respectively, from the pembrolizumab and cemiplimab trials. All 3 single-arm clinical trials had similar eligibility criteria (Supplementary Appendix B). KEYNOTE-629 $(\mathrm{N}=105)$ was used to inform the base case (Table 1) because it formed the basis of the pembrolizumab FDA license, was larger than the CARSKIN study $(\mathrm{N}=39)$, and was more similar to EMPOWER-CSCC-1 in that it was a global trial (CARSKIN was conducted in France). However, a separate scenario was performed to evaluate pembrolizumab efficacy based on the pooled trials. The patient characteristics were broadly comparable between EMPOWER-CSCC- 1 and KEYNOTE-629, with the exception of the primary site of disease $(68 \%$ of patients with the primary lesion on the head or neck in EMPOWER-CSCC-1 compared with $45 \%$ in KEYNOTE-629), severity of disease (46\% of patients had distant metastatic disease in EMPOWER-CSCC-1, compared with $55 \%$ in KEYNOTE-629), and prior treatment experience (34\% of patients in EMPOWER-CSCC-1 had received prior systemic therapy, compared with $87 \%$ in KEYNOTE-629).

Population-Adjusted Indirect Comparison. In studies that lack a control arm, such as EMPOWER-CSCC-1, it is challenging to distinguish which component of an observed outcome is attributable to treatment (ie, the treatment effect) and which part is attributable to disease characteristics (ie, prognostic factors). ${ }^{23}$ To account for differences in patient characteristics between EMPOWER-CSCC-1 and KEYNOTE-629, an unanchored population-adjusted indirect comparison was conducted, whereby IPD from EMPOWER-CSCC-1 were used to adjust baseline characteristics to align with the KEYNOTE-629 population, consistent with previously published methods. ${ }^{23,24}$ Two statistical methods were evaluated: a regression-based simulated treatment comparison (STC) and a matchingadjusted indirect comparison (MAIC) using propensity score weighting. Characteristics adjusted for in the analyses were age, disease stage, tumor location, tumor size 


\section{TABLE 2 Source Data for Cost-Effectiveness Model Inputs}

\begin{tabular}{|c|c|c|}
\hline Model input & Cemiplimab & Pembrolizumab \\
\hline \multicolumn{3}{|l|}{ Utilities } \\
\hline Preprogression utility ${ }^{24,47}$ & \multicolumn{2}{|c|}{0.820 (SE 0.009) } \\
\hline Postprogression utility ${ }^{24,47}$ & \multicolumn{2}{|c|}{0.776 (SE 0.015) } \\
\hline AE utility decrement (first cycle only) ${ }^{30,47-50, a}$ & \multicolumn{2}{|c|}{-0.001} \\
\hline \multicolumn{3}{|l|}{ Drug acquisition costs } \\
\hline Preprogression ${ }^{51}$ & $\begin{array}{c}350 \mathrm{mg} \text { Q3W } \\
350 \mathrm{mg} / 7 \mathrm{~mL} \text { vial; } \$ 9,236.50 / \text { vial }\end{array}$ & $\begin{array}{l}200 \mathrm{mg} \text { Q3W } \\
100 \mathrm{mg} / 4 \mathrm{~mL} \text { or } 25 \mathrm{mg} / \mathrm{mL} \text { single-dose vial; } \\
\$ 4,934.96 / \text { vial }\end{array}$ \\
\hline Postprogression (subsequent treatment) ${ }^{20}$ & \multicolumn{2}{|c|}{$\$ 4,000$ first month, $\$ 3,545$ subsequent months } \\
\hline \multicolumn{3}{|l|}{ Drug administration costs, USD } \\
\hline Preprogression ${ }^{20,52}$ & \multicolumn{2}{|c|}{143} \\
\hline Postprogression (subsequent treatment) ${ }^{20,52}$ & \multicolumn{2}{|c|}{329} \\
\hline \multicolumn{3}{|l|}{ Disease management costs, USD } \\
\hline Preprogression, 1 time ${ }^{53,54}$ & \multicolumn{2}{|c|}{54} \\
\hline Preprogression, per model cycle ${ }^{52,54}$ & \multicolumn{2}{|c|}{655} \\
\hline Postprogression, 1 time ${ }^{52,54}$ & 29 & 11 \\
\hline Postprogression, per model cycles ${ }^{52,54}$ & \multicolumn{2}{|c|}{1,132} \\
\hline Terminal care cost ${ }^{55,56}$ & \multicolumn{2}{|c|}{31,824} \\
\hline \multicolumn{3}{|l|}{ AE costs, ${ }^{47}$ USD } \\
\hline AE cost (first cycle only) $47,56,57, a, b$ & 345 & 713 \\
\hline \multicolumn{3}{|c|}{$\begin{array}{l}{ }^{a} A E \text { rates, utility decrements, and costs were sampled independently in the model. See Supplementary Appendix A (available in online article) for individual } \\
\text { parameters. } \\
\text { busing inverse variance. }\end{array}$} \\
\hline
\end{tabular}

(T stage), sex, Eastern Cooperative Oncology Group (ECOG) performance status (PS), previous systemic therapy, and previous radiotherapy. The adjusted estimates remained in favor of cemiplimab OS compared with pembrolizumab, using both the STC (hazard ratio $[\mathrm{HR}]=0.42,95 \% \mathrm{CI}=0.27-$ $0.65)$ and MAIC (HR $=0.52,95 \% \mathrm{CI}=0.34-0.80)$, compared with the unadjusted analysis $(\mathrm{HR}=0.42,95 \% \mathrm{CI}=0.27-$ 0.65). Cemiplimab also showed better PFS compared with pembrolizumab, in both the unadjusted $(\mathrm{HR}=0.53,95 \%$ $\mathrm{CI}=0.38-0.74)$ and adjusted (STC HR=0.58, 95\% CI $=0.42-$ 0.80 ; MAIC HR $=0.60,95 \% \mathrm{CI}=0.44-0.83$ ) comparisons..$^{23,24}$ The methods and results of the current analysis are provided in Supplementary Appendix C.

Despite the observed differences between EMPOWERCSCC-1 and KEYNOTE-629, the base-case analysis used the reported (naive) outcomes from both trials; however, the cemiplimab estimates from the adjusted comparisons were integrated into the cost-effectiveness analysis (CEA) as a scenario analysis.
Extrapolation of Survival in the Economic Model. Because a lifetime time horizon is required to evaluate expected outcomes in this advanced population, PFS and OS were extrapolated until all patients had progressed or died. Survival was extrapolated consistent with established recommendations from the National Institute for Health and Care Excellence (NICE) Decision Support Unit, ${ }^{27}$ which included an evaluation of the log cumulative hazard plots. The assessment of hazards showed violation of the proportional hazards assumption for PFS; as such, models were independently fitted to each treatment arm for both PFS and OS, which provided the most flexible approach and aligned with the nonrandomized single-arm trials informing the efficacy.

Alternative parametric distributions were fit to the IPD (cemiplimab, naive, and adjusted) and the reconstructed IPD (pembrolizumab). Alternative distributions included Weibull ( $\mathrm{p} 1=0)$, Gompertz ( $\mathrm{p} 1=1)$, log normal, log-logistic, and second-order fractional polynomials with powers 
defined as $\mathrm{p} 1=0$ or 1 , and power $\mathrm{p} 2=-1,-0.5,0,0.5$, or 1 . The fit of the models to the observed data was assessed using deviance information criteria; the tails of the PFS and OS functions were inspected visually to assess whether the extrapolations were plausible from a clinical and epidemiological perspective. Only distributions for PFS that were feasible in relation to the OS results were selected (ie, PFS $<$ OS). OS was capped using age-adjusted mortality rates for the US general population. ${ }^{33}$

For both PFS and OS, the log normal was selected as the best fitting distribution offering a clinically plausible extrapolation (until end of time horizon) that declined over time for both cemiplimab and pembrolizumab (Supplementary Appendix D, available in online article).

Treatment Duration. Cemiplimab is recommended for patients until either disease progression or unacceptable toxicity, which was the assumption for the base-case analysis. To account for the fact that PFS was greater than treatment duration (TD) in EMPOWER-CSCC-1, TD was based on PFS adjusted for TD using a PFS vs TD HR of 0.768 (95\% CI=0.584-1.009). This estimate from EMPOWERCSCC-1 censored patients who stopped treatment according to treatment stopping rules in the trial (Groups 1 and 2 [N=137] were not treated beyond 22.1 months [96 weeks]; Group $3[\mathrm{~N}=56]$ was not treated beyond 12.5 months [54 weeks]) to avoid underestimating TD.

Similarly, for pembrolizumab, a PFS vs TD HR of 0.841 (95\% CI $=0.105-6.734)$ was estimated from KEYNOTE-629 and applied to PFS to better approximate the treatment costs of pembrolizumab. ${ }^{30}$ However, unlike cemiplimab, the FDA license for pembrolizumab includes a treatment-stopping rule, whereby patients may receive pembrolizumab until disease progression or up to 24 months. ${ }^{34}$ Therefore, in addition to adjusting PFS to characterize TD, pembrolizumab treatment costs were stopped at 24 months. The cessation of treatment at 24 months limited the treatment costs as compared with cemiplimab, where patients were treated until progression. Further, the treatment benefit for pembrolizumab was assumed to continue over the full model horizon despite treatment cessation at 24 months. Both assumptions were evaluated in the scenario analyses.

Adverse Events. Only grade 3 or 4 adverse events (AEs) associated with cemiplimab or pembrolizumab were included in the model if reported in at least $5 \%$ of the patients in the clinical trials, as these AEs were expected to incur higher costs and have a greater impact on health-related quality of life. This approach is consistent with the previous CEA of cemiplimab. ${ }^{24}$ Estimates for the grade 3 or 4 AEs are summarized in Supplementary Appendix A, alongside the unit costs and utility decrements for each event.

Utilities, Cost, and Resource Use. Model inputs and assumptions informing utilities, resource use, and costs are detailed in Table 2 and described in Supplementary Appendix A.

\section{ANALYSES}

For the base case, point estimates informed the incremental discounted total cost, incremental discounted QALYs, and incremental cost-effectiveness ratio (ICER).

One-way sensitivity analysis, summarized in a tornado diagram, and probabilistic sensitivity analysis (PSA), presented as a cost-effectiveness acceptability curve, were conducted consistent with Konidaris et al. ${ }^{24}$

Scenario analyses were conducted to evaluate key assumptions in the analysis related to extrapolation of survival, TD, and AEs.

To assess assumptions related to the modeling of survival and efficacy, the following scenarios were evaluated: alternative distributions for cemiplimab and pembrolizumab OS (Weibull, log logistic, Gompertz); cemiplimab-adjusted on the basis of patient characteristics in KEYNOTE-629 using an STC (Methods and Results in Supplementary Appendix C); and treatment effects for pembrolizumab derived from pooled KEYNOTE-629 and CARSKIN studies.

Consistent with the FDA licenses for pembrolizumab and cemiplimab, the base case assumed that cemiplimab was continued until disease progression, whereas pembrolizumab treatment was stopped at 24 months. This assumption favors pembrolizumab, as not only is the treatment benefit assumed to continue long after treatment cessation, but treatment costs are also capped at 24 months. The following scenario analyses evaluated assumptions related to TD: the effect (ie, hazard) of pembrolizumab was assumed to stop at 24 months at which point it was set to equal hazard of historical SOC (prior to the availability of cemiplimab) at 24 months ${ }^{24}$; pembrolizumab treatment was assumed to continue until progression, consistent with the cemiplimab license; the same PFS vs HR adjustment was applied to both treatment arms (PFS vs TD HR from EMPOWER-CSCC-1 of 0.768).

Finally, since only one grade 3 or $4 \mathrm{AE}$ was identified as occurring in at least $5 \%$ of the patients in EMPOWERCSCC-1 and KEYNOTE-629 trials (anemia), the impact of lowering the threshold to at least $3 \%$ occurrence was evaluated. 


\section{TABLE 3 Summary of Base-Case, Probabilistic, and Scenario Analyses}

\begin{tabular}{|c|c|c|c|c|c|c|c|}
\hline \multirow[b]{2}{*}{ Treatment } & \multicolumn{3}{|c|}{ Total (discounted) } & \multicolumn{3}{|c|}{ Incremental (discounted) } & \multirow[b]{2}{*}{ ICER, USD } \\
\hline & Costs, USD & LYs & QALYs & Costs, USD & LYs & QALYs & \\
\hline \multicolumn{8}{|l|}{ Base-case results } \\
\hline Cemiplimab & 683,061 & 7.39 & 5.90 & - & - & - & - \\
\hline Pembrolizumab & 320,817 & 3.95 & 3.12 & 362,244 & 3.44 & 2.78 & 130,329 \\
\hline \multicolumn{8}{|l|}{ Probabilistic analysis } \\
\hline Cemiplimab & 683,365 & 7.37 & 5.87 & - & - & - & - \\
\hline Pembrolizumab & 319,633 & 3.97 & 3.13 & 363,733 & 3.40 & 2.74 & 132,589 \\
\hline \multicolumn{8}{|l|}{ Scenario analysis } \\
\hline \multicolumn{8}{|l|}{ Weibull for OS } \\
\hline Cemiplimab & 636,251 & 6.57 & 5.26 & - & - & - & - \\
\hline Pembrolizumab & 277,055 & 3.21 & 2.54 & 359,196 & 3.37 & 2.72 & 132,073 \\
\hline \multicolumn{8}{|l|}{ Log logistic for OS } \\
\hline Cemiplimab & 660,648 & 7.01 & 5.60 & - & - & - & - \\
\hline Pembrolizumab & 310,626 & 3.78 & 2.98 & 350,022 & 3.23 & 2.61 & 133,949 \\
\hline \multicolumn{8}{|l|}{ Gompertz for OS } \\
\hline Cemiplimab & 722,418 & 8.07 & 6.42 & - & - & - & - \\
\hline Pembrolizumab & 469,176 & 6.46 & 5.07 & 253,242 & 1.60 & 1.35 & 187,374 \\
\hline \multicolumn{8}{|c|}{ Cemiplimab STC adjusted } \\
\hline Cemiplimab & 680,287 & 7.83 & 6.22 & - & - & - & - \\
\hline Pembrolizumab & 320,817 & 3.95 & 3.12 & 359,470 & 3.88 & 3.10 & 115,909 \\
\hline \multicolumn{8}{|c|}{ Pooled pembrolizumab studies } \\
\hline Cemiplimab & 684,563 & 7.39 & 5.89 & - & - & - & - \\
\hline Pembrolizumab & 313,660 & 3.89 & 3.09 & 370,903 & 3.50 & 2.81 & 132,158 \\
\hline
\end{tabular}

Pembrolizumab treatment effect (hazard) switched to that of historical SOC at 24 months

\begin{tabular}{l|c|c|c|c|c|c|c}
\hline Cemiplimab & 683,061 & 7.39 & 5.90 & - & - & - & - \\
\hline Pembrolizumab & 268,305 & 3.37 & 2.68 & 414,756 & 4.02 & 3.21 & 129,063 \\
\hline
\end{tabular}

Pembrolizumab treatment until progression

\begin{tabular}{l|c|c|c|c|c|c|c}
\hline Cemiplimab & 683,061 & 7.39 & 5.90 & - & - & - & - \\
\hline Pembrolizumab & 359,130 & 3.95 & 3.12 & 323,931 & 3.44 & 2.78 & 116,545 \\
\hline
\end{tabular}

PFS vs TD HR from EMPOWER-CSCC-1 applied to pembrolizumab

\begin{tabular}{l|c|c|c|c|c|c|c}
\hline Cemiplimab & 683,061 & 7.39 & 5.90 & - & - & - & - \\
\hline Pembrolizumab & 311,916 & 3.95 & 3.12 & 371,145 & 3.44 & 2.78 & 133,531 \\
\hline
\end{tabular}

Grade 3 or 4 AEs occurring in $\geq 3 \%$ of patients in either treatment arm

\begin{tabular}{l|c|c|c|c|c|c|c}
\hline Cemiplimab & 684,246 & 7.39 & 5.89 & - & - & - & - \\
\hline Pembrolizumab & 321,111 & 3.95 & 3.12 & 363,135 & 3.44 & 2.78 & 130,687 \\
\hline
\end{tabular}

$A E=$ adverse event; $H R=$ hazard ratio; ICER = incremental cost-effectiveness ratio; $L Y=$ life-year; $O S=$ overall survival; $P F S=$ progression-free survival; $Q A L Y=$ quality-adjusted life-year; $S O C=$ standard of care; $S T C=$ simulated treatment comparison; $T D=$ treatment duration; USD $=U S$ dollars. 


\section{FIGURE 1 Tornado Plot of Base-Case 1-Way Sensitivity Analysis}

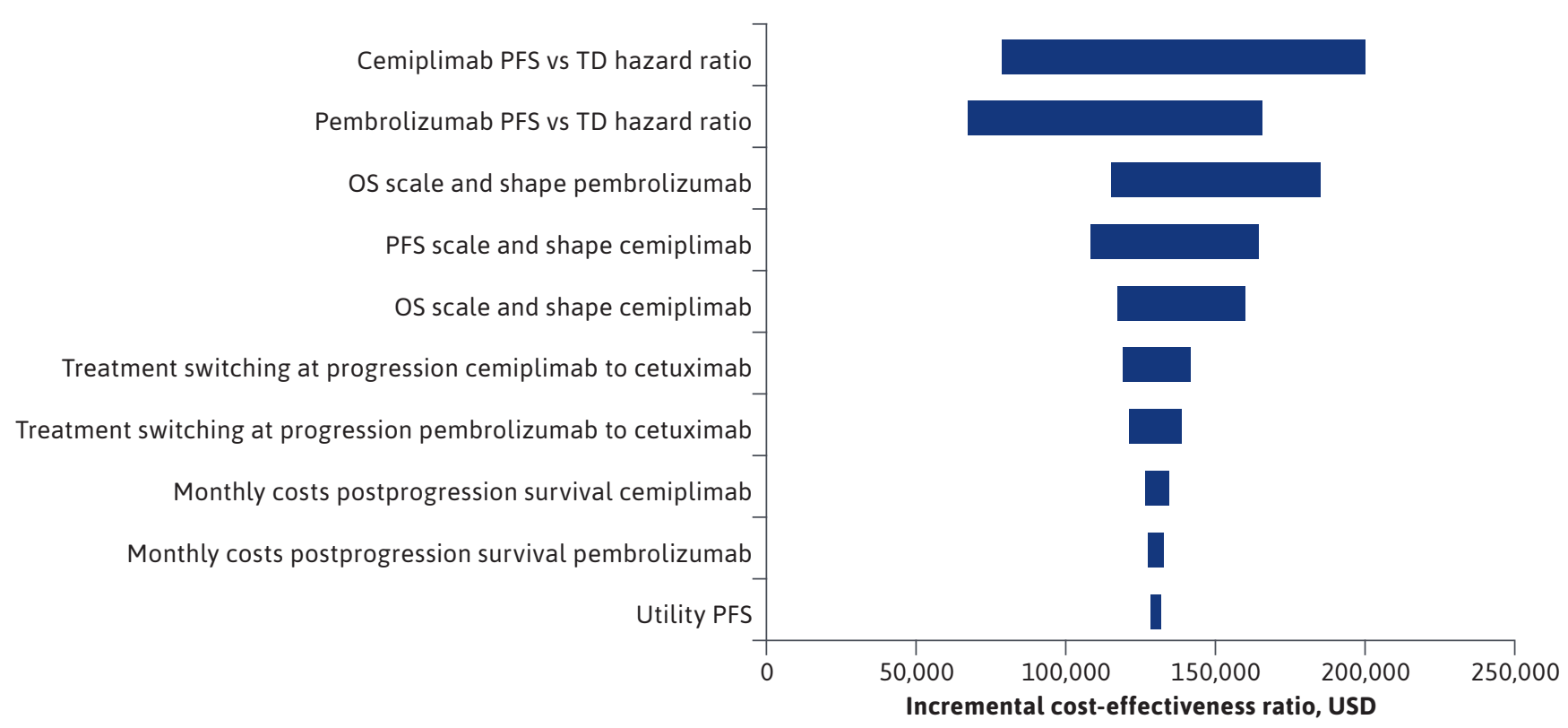

OS=overall survival; $P F S=$ progression free survival; $T D=$ treatment duration; USD = US dollars

\section{Results}

\section{BASE-CASE ANALYSIS}

Treatment with cemiplimab was associated with an additional 3.44 discounted LYs compared with pembrolizumab (7.39 vs 3.95, respectively) over the model horizon, reflecting increased time of modeled patients in both the preprogression (3.57 vs 1.03) and postprogression (3.82 vs 2.92) health states. Consequently, patients treated with cemiplimab accrued an additional 2.78 QALYs vs pembrolizumab over the time horizon of the model, with cemiplimab and pembrolizumab accruing 5.90 QALYs vs 3.12 QALYs, respectively. Driven by the increased time spent in the progression-free state by patients treated with cemiplimab, total discounted costs in the base case (Table 3) for cemiplimab were $\$ 362,244$ higher than for pembrolizumab $(\$ 683,061$ vs $\$ 320,817$, respectively). In terms of value, the base-case analysis found cemiplimab treatment resulted in an ICER of $\$ 130,329 / Q A L Y$ vs pembrolizumab, well below the willingness-to-pay (WTP) threshold of \$150,000/QALY in the United States for nonrare diseases. Disaggregated results of CEA are provided in Supplementary Appendix E (available in online article).

\section{ONE-WAY SENSITIVITY ANALYSIS}

One-way sensitivity analysis indicated that the parameter with the greatest impact on the ICER was the TD adjustment applied to cemiplimab PFS (Figure 1). Beyond TD, the parameters with the greatest impact on the ICER were the TD adjustment applied to pembrolizumab PFS, the OS parameters for pembrolizumab, and the PFS and OS parameters for cemiplimab.

\section{PROBABILISTIC SENSITIVITY ANALYSIS}

The PSA resulted in a mean ICER of $\$ 132,589$ for cemiplimab vs pembrolizumab (Table 3), which was consistent with the deterministic base-case result. Although the probability of cemiplimab being cost-effective at a WTP threshold of $\$ 100,000 /$ QALY is low $(8 \%$, Figure 2$)$, cemiplimab has a $71 \%$ probability of being cost-effective when compared with pembrolizumab at a WTP threshold of \$150,000/QALY.

\section{SCENARIO ANALYSIS}

Assuming alternative distributions for cemiplimab and pembrolizumab OS (Weibull, log logistic, Gompertz) resulted in ICERs of \$132,073/QALY, \$133,949/QALY, and \$187,374/ QALY, respectively (Table 3). The distributions for the base case offer a better fit compared with the other distributions when considering both the statistical fit and tail end of the 


\section{FIGURE 2 Base-Case Probabilistic Sensitivity Analysis: Cost-Effectiveness Acceptability Curves of Cemiplimab and Pembrolizumab (1,000 Iterations)}

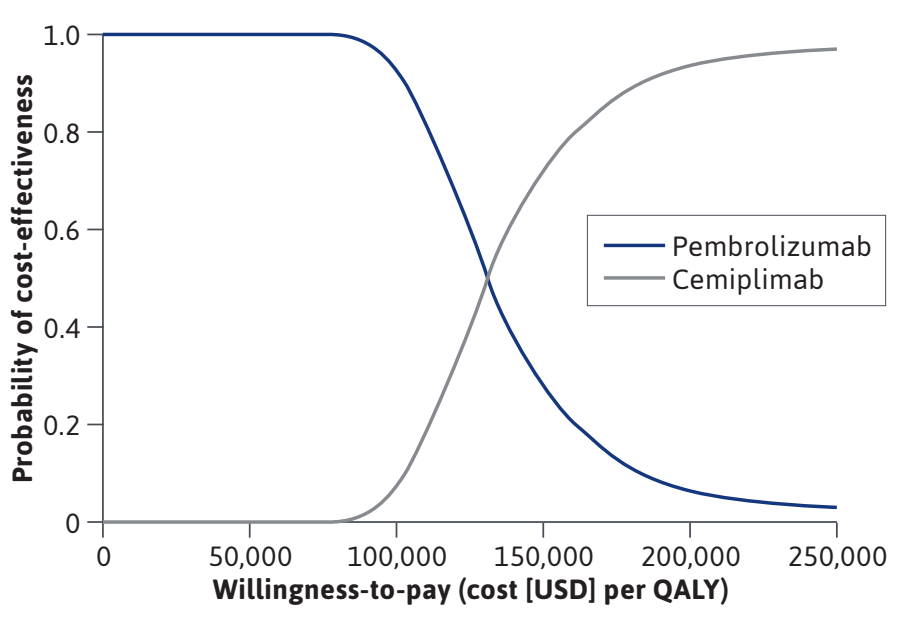

$Q A L Y=$ quality-adjusted life-year; $U S D=U S$ dollars

extrapolation (ie, clinical plausibility). Results of both the naive (unadjusted) and adjusted comparisons were favorable for cemiplimab. Cemiplimab adjusted (STC) based on the patient characteristics as observed in KEYNOTE-629 was included in the model, shifting the ICER in favor of cemiplimab to $\$ 115,909 / \mathrm{QALY}$. The change was driven by a slight lift in extrapolated cemiplimab OS when modeling the hazards generated from the STC, increasing postprogression QALYs for cemiplimab compared with the base case, while pembrolizumab survival, QALYs, and costs remained consistent with the base case.

Changing the estimate for the efficacy of pembrolizumab from KEYNOTE-629 to the pooled estimates from KEYNOTE-629 and CARSKIN trials had minimal impact on the ICER (\$132,158/QALY) given the small sample size of CARSKIN ( $=39$ compared with $\mathrm{N}=105$ in KEYNOTE-629). Halting the treatment effect of pembrolizumab at 24 months (ie, switching the hazards in the pembrolizumab arm to that of historical SOC at 24 months) had a negligible impact on the results: $\$ 129,063$ for new scenario vs $\$ 130,329$ base case. However, assuming treatment until progression for pembrolizumab (rather than a 24-month treatment-stopping rule) shifted the ICER in favor of cemiplimab to $\$ 116,545 / \mathrm{QALY}$, due to an increase in total preprogression drug acquisition and administration costs for pembrolizumab from $\$ 114,521$ to $\$ 152,288$. Applying the PFS vs TD HR from EMPOWER-CSCC-1 to the pembrolizumab arm only marginally affected the ICER (\$133,531/QALY). Similarly, the ICER was not sensitive to the threshold for grade 3 or 4 AEs (ie, changing to at least $3 \%$ resulted in ICER $\$ 130,687 /$ QALY).

\section{Discussion}

This CEA of cemiplimab vs pembrolizumab, the newest PD-1 receptor inhibitor approved treatment for advanced CSCC, suggests that cemiplimab is a cost-effective treatment for advanced CSCC. The analysis was conducted according to best practice guidelines and based on a cohort model with a "partitioned survival" structure. ${ }^{24,27}$ Data to inform the efficacy and safety of cemiplimab in the analysis were from EMPOWER-CSCC-1, which represents the most extensive cohort of advanced CSCC patients published to date.

To inform the efficacy of pembrolizumab, outcomes from the pivotal trial KEYNOTE-629 were identified based on a systematic literature review. The EMPOWER-CSCC-1 and KEYNOTE-629 trials were both single-arm clinical trials evaluating patients with unresectable laCSCC or mCSCC (ECOG PS 0-1), who had not received prior therapy with an anti-PD-1/PD-ligand 1, or systemic antitumor treatment within 4 weeks of study. The populations between the trials were broadly comparable; however, patients in KEYNOTE-629 had more severe disease and were more heavily pretreated, differences that were evaluated by means of an unanchored population-adjusted indirect comparison.

Although median survival for cemiplimab was not reached, predicted survival at 1 year (83\%), 2 years $(73 \%)$, and 5 years (56\%) suggests a more favorable life expectancy for patients treated with cemiplimab over predicted pembrolizumab (64\%, $48 \%$, and $28 \%$, respectively), which led to an incremental gain of 3.44 LYs (discounted) and an ICER of $\$ 130,329 / Q A L Y$ in the base case. In the absence of a headto-head randomized controlled trial comparing cemiplimab to pembrolizumab, there is a risk that treatment effect estimates based on the naive comparison (unadjusted) are biased due to between-study differences. Hence, an unanchored population-adjusted indirect comparison for cemiplimab vs pembrolizumab was performed for OS and PFS using an STC and MAIC. These methods adjust outcomes based on between-study differences in baseline characteristics. Although patients in KEYNOTE-629 had more severe disease and were more heavily pretreated, estimates of OS and PFS were in favor of cemiplimab compared with pembrolizumab in both the unadjusted (naive) and adjusted (STC and MAIC) comparisons. When included in the economic model, the predicted survival estimates for cemiplimab from the STC resulted in a more favorable 
cost-effectiveness estimate for cemiplimab (\$115,909/ QALY), driven by a slight lift in cemiplimab OS when applying the STC due to the extrapolation fit; thus, this scenario was not used as the base-case analysis.

Results were most sensitive to changes in TD parameters based on the 1-way sensitivity analysis. Cemiplimab costs were based on treat to progression with no stopping rule. In contrast, treatment with pembrolizumab was assumed to be stopped at 24 months in line with the FDA license, although the treatment benefit of pembrolizumab was assumed to continue over the time horizon of the model. Recent technology appraisals of immunotherapies evaluated by NICE have critiqued the application of a lifetime treatment benefit when a treatment stopping rule is applied ${ }^{35-39}$ and suggest a cessation of treatment benefit following treatment discontinuation, noting considerable uncertainty as to the size and duration of benefit due to the incomplete follow-up. ${ }^{35,37}$ This is important given estimates from this study suggest most of the benefits for pembrolizumab are accrued postprogression, in contrast to cemiplimab where benefits are mostly attributed to gain in preprogression. Further, it could be speculated that there are unlikely to be differences in the way the 2 treatments are used in clinical practice given they are both PD-1 inhibitors. If we assume the same treatment approach for pembrolizumab as cemiplimab (ie, treatment to progression with no treatment cap) the ICER moved in favor of cemiplimab to $\$ 116,545 / \mathrm{QALY}$.

One-way sensitivity analysis also identified OS and PFS as the key drivers of results for both cemiplimab and pembrolizumab. As always, there is structural uncertainty regarding the most appropriate model to extrapolate longterm PFS and OS. Therefore, it will be important to reassess cost-effectiveness in the future based on longer follow-up from EMPOWER-CSCC-1 (median follow-up of 15.7 months) and, in particular, for KEYNOTE-629 (median follow-up 11.4 months). Using a pooled estimate from both KEYNOTE-629 in combination with CARSKIN trial, which has a longer follow-up (median follow-up 22.4 months), had minimal impact on the ICER $(\$ 132,158 /$ QALY).

On the other hand, cemiplimab is presently priced at an $8 \%$ discount relative to pembrolizumab in the United States. Thus, structural uncertainty around the long-term extrapolation notwithstanding and given that treatment costs are largely informed by the duration of PFS, our finding that cemiplimab is the cost-effective option is likely to hold under a wide range of extrapolation scenarios. In the extreme case where cemiplimab had identical OS and PFS as pembrolizumab, it would remain the cost-effective option by virtue of its lower list price.
The Institute for Clinical and Economic Review recommends a threshold of $\$ 100,000$ to $\$ 150,000 /$ QALY, with the upper range recommended by the World Health Organization. ${ }^{40}$ The results of the current base-case analysis fall below a \$150,000/QALY threshold, and the PSA suggests a $71 \%$ probability that cemiplimab will be a costeffective treatment choice compared with pembrolizumab at a WTP threshold of $\$ 150,000 / Q A L Y$. Results of scenario analyses testing key assumptions informing TD and clinical effectiveness showed the ICER to be relatively robust with only 1 scenario falling above the \$150,000/QALY WTP threshold.

The present results also underscore the previous findings on the cost-effectiveness of cemiplimab from a US payer perspective ${ }^{23,24}$ based on an interim cut of cemiplimab clinical trial data (NCT02760498, median follow-up of 11.9 months) and compared with historical SOC (ie, chemotherapy and EGFR inhibitors). ${ }^{14-17,41}$ The prior evaluation indicated substantial clinical and economic value of cemiplimab in patients with advanced CSCC relative to historically used treatments, driven by favorable OS and PFS for cemiplimab compared with the treatments that collectively comprised SOC prior to the introduction of immunotherapy for advanced CSCC.

Approximately 10,000 patients in the United States are expected to suffer from advanced CSCC. ${ }^{1,42}$ Until recently, the treatment pathway of patients with advanced CSCC had not been well established. Before cemiplimab and pembrolizumab, there were no FDA-approved systemic therapies for the treatment of advanced $\operatorname{CSCC}^{9,34,43}$ and no treatment recommendations for advanced CSCC.9,44 Traditionally, clinicians relied on treatments with limited supporting evidence based on small patient populations, in some cases from other populations such as head and neck squamous cell carcinoma. ${ }^{9,45}$ With the recent availability of immunotherapies and given the demonstrated clinical superiority of cemiplimab compared with historical SOC, ${ }^{23,24}$ it is anticipated that the treatment pathway for patients with advanced CSCC will prioritize treatment with immunotherapies over other systemic therapies. Results from these analyses suggest that cemiplimab offers a cost-effective treatment for patients with advanced CSCC compared with pembrolizumab.

\section{LIMITATIONS}

This analysis is not without limitations. There were no headto-head cemiplimab vs pembrolizumab trials in patients with advanced CSCC to inform relative treatment effects in the cost-effectiveness model; thus, the naive comparisons may be at risk of bias. Given the differences between the populations included in EMPOWER-CSCC-1 and KEYNOTE-629 
in terms of primary site of disease, severity of disease, and prior treatment experience, population-adjusted indirect comparisons were conducted to facilitate a more accurate comparison between the 2 studies.

Although the adjusted estimates remained in favor of cemiplimab for both PFS and OS, ${ }^{46}$ it is unclear whether any unknown or unmeasured prognostic factors of survival missing from the models may have influenced the outcomes of interest. More broadly, there may be residual uncertainty in the analysis driven by limited information, small sample size, and incomplete follow-up of the clinical trials available for this rare disease.

The result of the analyses should be cautiously interpreted in the absence of a head-to-head randomized controlled trial comparing cemiplimab to pembrolizumab.

\section{Conclusions}

Our analysis shows that compared with pembrolizumab, cemiplimab is a cost-effective treatment for patients with advanced CSCC, suggesting significant relative clinical and economic value to health care payers in the United States.

\section{DISCLOSURES}

This study was supported by Regeneron Pharmaceuticals, Inc., and Sanofi. Paul, Cope, Keeping, Mojebi, and Ayers are employees of PRECISIONheor, which received funding to produce this work. Chen, Kuznik, and $\mathrm{Xu}$ are employees and stockholders of Regeneron Pharmaceuticals, Inc. Sasane is an employee and stockholder of Sanofi, Inc. Konidaris, Atsou, and Guyot are employees of Sanofi, Inc. The authors were responsible for all content and editorial decisions and received no honoraria related to the development of this publication.

\section{ACKNOWLEDGMENTS}

Writing support was provided by Gauri Saal, MA, and Kate Carolan, PhD, of Prime Global (Knutsford, UK) and was funded by Regeneron Pharmaceuticals, Inc., and Sanofi Genzyme.

\section{REFERENCES}

1. Rogers HW, Weinstock MA, Feldman SR, Coldiron BM. Incidence estimate of nonmelanoma skin cancer (keratinocyte carcinomas) in the U.S. population, 2012. JAMA Dermatol. 2015;151(10):1081-86.

2. Lucas RM, McMichael T, Smith W, Armstrong B. Solar ultraviolet radiation: global burden of disease from solar ultraviolet radiation. Environmental Burden of Disease Series, no. 13. World Health Organization. 2006. Accessed June 16, 2020. https:// apps.who.int/iris/bitstream/handle $/ 10665 / 43505 / 9241594403$ eng. pdf? sequence $=1 \&$ is Allowed $=\mathrm{y}$

3. Ducroux E, Martin C, Bouwes Bavinck JN, et al. Risk of aggressive skin cancers after kidney retransplantation in patients with previous posttransplant cutaneous squamous cell carcinomas: a retrospective study of 53 cases. Transplantation. 2017;101(4):e133-41.

4. Corchado-Cobos R, García-Sancha N, González-Sarmiento R, Pérez-Losada J, Cañueto J. Cutaneous squamous cell carcinoma: from biology to therapy. Int J Mol Sci. 2020;21(8):2956.

5. Lindelof B, Jarnvik J, Ternesten-Bratel A, Granath F, Hedblad MA. Mortality and clinicopathological features of cutaneous squamous cell carcinoma in organ transplant recipients: a study of the Swedish cohort. Acta Derm Venereol. 2006;86(3):219-22.

6. Manyam BV, Garsa AA, Chin RI, et al. A multi-institutional comparison of outcomes of immunosuppressed and immunocompetent patients treated with surgery and radiation therapy for cutaneous squamous cell carcinoma of the head and neck. Cancer. 2017;123(11):2054-60.
7. McLaughlin EJ, Miller L, Shin TM, et al. Rate of regional nodal metastases of cutaneous squamous cell carcinoma in the immunosuppressed patient. Am J Otolaryngol. 2017;38(3):325-28.

8. Velez NF, Karia PS, Vartanov AR, Davids MS, Brown JR, Schmults CD. Association of advanced leukemic stage and skin cancer tumor stage with poor skin cancer outcomes in patients with chronic lymphocytic leukemia. JAMA Dermatol. 2014;150(3):280-87.

9. Stratigos AJ, Garbe C, Lebbe C, et al. Diagnosis and treatment of invasive squamous cell carcinoma of the skin: European consensus-based interdisciplinary guideline. Eur J Cancer. 2015;51(14):1989-2007.

10. Schmults CD, Karia PS, Carter JB, Han J, Qureshi AA. Factors predictive of recurrence and death from cutaneous squamous cell carcinoma: a 10-year, single-institution cohort study. JAMA Dermatol. 2013;149(5):541-47.

11. Karia PS, Han J, Schmults CD.

Cutaneous squamous cell carcinoma: estimated incidence of disease, nodal metastasis, and deaths from disease in the United States, 2012. J Am Acad Dermatol. 2013;68(6):957-66.

12. Bossi P, Cavalieri S, Perrone F, et al. Efficacy and safety of single agent panHER inhibitor dacomitinib in locally advanced unresectable or metastatic skin squamous cell cancer (sSCC). J Clin Oncol. 2017;35(15 Suppl):9543-43.

13. Sun L, Chin RI, Gastman B, et al. Association of disease recurrence with survival outcomes in patients with cutaneous squamous cell carcinoma of the head and neck treated with multimodality therapy. JAMA Dermatol. 2019;155(4):442-47.

14. Jarkowski A, 3rd., Hare R, Loud P, et al. Systemic therapy in advanced cutaneous squamous cell carcinoma (CSCC): the Roswell Park Experience and a review of the literature. Am J Clin Oncol. 2016;39(6):545-48 
15. Gold KA, Kies MS, William WN, Jr., Johnson FM, Lee JJ, Glisson BS. Erlotinib in the treatment of recurrent or metastatic cutaneous squamous cell carcinoma: a single-arm phase 2 clinical trial. Cancer. 2018;124(10):2169-73.

16. William WN, Jr., Feng L, Ferrarotto R, et al. Gefitinib for patients with incurable cutaneous squamous cell carcinoma: a single-arm Phase II clinical trial. J Am Acad Dermatol. 2017;77(6):1110-13 e2.

17. Maubec E, Petrow P, Scheer-Senyarich I, et al. Phase II study of cetuximab as firstline single-drug therapy in patients with unresectable squamous cell carcinoma of the skin. J Clin Oncol. 2011;29(25):3419-26.

18. Picard A, Pedeutour F, Peyrade F, et al. Association of oncogenic mutations in patients with advanced cutaneous squamous cell carcinomas treated with cetuximab. JAMA Dermatol. 2017;153(4):291-98

19. Foote MC, McGrath M, Guminski A, et al. Phase II study of single-agent panitumumab in patients with incurable cutaneous squamous cell carcinoma. Ann Oncol. 2014;25(10):2047-52.

20. Cowey CL, Robert NJ, Espirito JL, et al. Clinical outcomes among unresectable, locally advanced, and metastatic cutaneous squamous cell carcinoma patients treated with systemic therapy. Cancer Med. 2020;9(20):7381-87.

21. Montaudié H, Viotti J, Combemale P, et al. Cetuximab is efficient and safe in patients with advanced cutaneous squamous cell carcinoma: a retrospective, multicentre study. Oncotarget. 2020;11(4);378-85.

22. Libtayo. Prescribing information. Regeneron Pharmaceuticals, Inc., 2021. Accessed February 25, 2021. https://www. accessdata.fda.gov/drugsatfda_docs/ label/2021/761097s007lbl.pdf

23. Keeping S, Xu Y, Chen C, et al. Comparative efficacy of cemiplimab versus other systemic treatments for advanced cutaneous squamous cell carcinoma. Future Oncol. 2020;17(5):611-27.
24. Konidaris G, Paul E, Kuznik A, et al. Assessing the value of cemiplimab for adults with advanced cutaneous squamous cell carcinoma: a cost-effectiveness analysis. Value Health. 2021;24(3):377-87.

25. Keytruda. Prescribing information. Merck and Co., Inc., 2020. Accessed June 29, 2020. https://www.accessdata.fda.gov/drugsatfda docs/ label/2020/125514s068lbl.pdf

26. Goeree R, Villeneuve J, Goeree J, Penrod JR, Orsini L, Tahami Monfared AA. Economic evaluation of nivolumab for the treatment of second-line advanced squamous NSCLC in Canada: a comparison of modeling approaches to estimate and extrapolate survival outcomes. J Med Econ. 2016;19(6):630-44

27. Latimer N. NICE DSU technical support document 14: Undertaking survival analysis for economic evaluations alongside clinical trials - extrapolation with patient-level data. June 2011. Updated March 2013. Accessed July 16, 2020. http://nicedsu.org.uk/wp-content/ uploads/2016/03/NICE-DSU-TSDSurvival-analysis.updated-March-2013. v2.pdf

28. Sanders GD, Neumann PJ, Basu A, et al. Recommendations for conduct, methodological practices, and reporting of cost-effectiveness analyses: second panel on cost-effectiveness in health and medicine. JAMA. 2016;316(10):1093-103.

29. Rischin D, Khushalani NI, Schmults CD, et al. Phase II study of cemiplimab in patients (pts) with advanced cutaneous squamous cell carcinoma (CSCC): longer follow-up. J Clin Oncol. 2020;38(15 Suppl):10018.

30. Grob JJ, Gonzalez R, Basset-Seguin N, et al. Pembrolizumab monotherapy for recurrent or metastatic cutaneous squamous cell carcinoma: a single-arm Phase II trial (KEYNOTE-629). J Clin Oncol. 2020;38(25):2916-25.

31. Maubec E, Boubaya M, Petrow P, et al. Phase II study of pembrolizumab as first-line, single-drug therapy for patients with unresectable cutaneous squamous cell carcinomas. J Clin Oncol. 2020;38(26):3051-61.
32. Guyot P, Ades A, Ouwens MJ, Welton NJ. Enhanced secondary analysis of survival data: reconstructing the data from published Kaplan-Meier survival curves. BMC Med Res Methodol. 2012;12(1):9.

33. Centers for Disease Control and Prevention. National vital statistics system, mortality data. 2017. Accessed July 20, 2020. https://www.cdc.gov/nchs/ nvss/deaths.htm

34. US Food and Drug Administration. FDA approves pembrolizumab for cutaneous squamous cell carcinoma. June 24, 2020. Accessed August 2020. https://www.fda.gov/drugs/ drug-approvals-and-databases/ fda-approves-pembrolizumab-cutaneoussquamous-cell-carcinoma

35. National Institute for Health and Care Excellence. Single technology appraisal 428: pembrolizumab for treating PD-L1positive non-small-cell lung cancer after chemotherapy. September 12, 2017. Accessed August 2020. https://www.nice. org.uk/guidance/ta428

36. National Institute for Health and Care Excellence. Single technology appraisal 490: nivolumab for treating recurrent or metastatic squamous-cell carcinoma of the head and neck after platinum-based chemotherapy. 2016. Accessed August 2019. https://www.nice.org.uk/guidance/ ta490/documents/committee-papers-2

37. National Institute for Health and Care Excellence. Single technology appraisal 531: pembrolizumab for untreated PD-L1positive metastatic non-small-cell lung cancer. July 18, 2018. Accessed November 4, 2019. https://www.nice.org.uk/ guidance/ta531/history

38. National Institute for Health and Care Excellence. Single technology appraisal 530: nivolumab for treating metastatic or unresectable urothelial cancer after platinum-based chemotherapy. July 14, 2018. Accessed November 2019. https://www. nice.org.uk/guidance/ta530 
39. National Institute for Health and Care Excellence. Technology appraisal guidance [TA519]: Pembrolizumab for treating locally advanced or metastatic urothelial carcinoma after platinum-containing chemotherapy. April 25, 2018. Accessed September 28, 2020. https://www.nice. org.uk/guidance/ta519/resources/ pembrolizumab-for-treating-locallyadvanced-or-metastatic-urothelial-carcinoma-after-platinumcontaining-chemotherapy-pdf-82606788406213

40. World Health Organization. Report of the commission on macroeconomics and health: macroeconomics and health: investing in health for economic development. December 20, 2001. Accessed February 2017. http://apps.who.int/iris/ bitstream/10665/42435/1/924154550X. pdf

41. Peyrade F, Kogay M, Viotti J, et al. Cetuximab in patients with unresectable cutaneous squamous cell carcinoma is safe and effective: a real-life analysis. Ann Oncol. 2018;29(Suppl 8):viii442-66.

42. Clayman GL, Lee JJ, Holsinger FC, et al. Mortality risk from squamous cell skin cancer. J Clin Oncol. 2005;23(4):759-65.

43. US Food and Drug Administration. FDA approves cemiplimab-rwlc for metastatic or locally advanced cutaneous squamous cell carcinoma. January 18, 2019. Accessed November 2019. https://www.fda.gov/ drugs/drug-approvals-and-databases/ fda-approves-cemiplimab-rwlc-metastatic-or-locally-advanced-cutaneoussquamous-cell-carcinoma

44. Cranmer LD, Engelhardt C, Morgan SS. Treatment of unresectable and metastatic cutaneous squamous cell carcinoma. Oncologist. 2010;15(12):1320-28.

45. Palyca P, Koshenkov VP, Mehnert JM. Developments in the treatment of locally advanced and metastatic squamous cell carcinoma of the skin: a rising unmet need. Am Soc Clin Oncol Educ Book. 2014:e397-404

46. Phillippo DM, Ades AE, Dias S, Palmer S, Abrams KR, Welton NJ. Methods for population-adjusted indirect comparisons in health technology appraisal. Med Decis Making. 2018;38(2):200-11.
47. Regeneron Pharmaceuticals Inc/ Sanofi. Individual patient data for the Phase 2 cemiplimab trial (R2810ONC-1540). Data on file. 2019.

48. Nafees B, Stafford M, Gavriel S, Bhalla S, Watkins J. Health state utilities for non small cell lung cancer. Health Qual Life Outcomes. 2008;6:84.

49. National Institute for Health and Care Excellence. Single technology appraisal 517: avelumab for treating metastatic Merkel cell carcinoma. April 21, 2021. Accessed July 2019. https://www.nice.org. uk/guidance/ta517

50. Doyle S, Lloyd A, Walker M. Health state utility scores in advanced nonsmall cell lung cancer. Lung Cancer. 2008;62(3):374-80.

51. ProspectoRx. February 2021, US drug prices. Data on file. 2021.

52. Centers for Medicare and Medicaid Services. Physician fee schedule search. 2020, Accessed March 17, 2020. https:// www.cms.gov/apps/physician-fee-schedule/overview.aspx

53. Centers for Medicare and Medicaid Services. Clinical laboratory fee schedule. 2020. Accessed July 2020. https://www. cms.gov/Medicare/Medicare-Fee-forService-Payment/ClinicalLabFeeSched/ index

54. Regeneron Pharmaceuticals Inc/ Sanofi. Expert opinion. Data on file. 2019.

55. Chastek B, Harley C, Kallich J, Newcomer L, Paoli CJ, Teitelbaum AH. Health care costs for patients with cancer at the end of life. J Oncol Pract. 2012;8(6):75s-80s.

56. United States Bureau of Labor Statistics. Consumer Price Index. 2020. Accessed July 17, 2020. https://www.bls. gov/cpi/

57. Agency for Healthcare Research and Quality. Healthcare cost and utilization project (HCUP). 2021. Accessed July 2020. https://hcupnet.ahrq.gov/

58. O'Mahony JF, Newall AT, van Rosmalen J. Dealing with time in health economic evaluation: methodological issues and recommendations for practice. Pharmacoeconomics. 2015;33(12):1255-68.
59. Longworth L, Yang Y, Young T, et al. Use of generic and condition-specific measures of health-related quality of life in NICE decision-making: a systematic review, statistical modelling and survey. Health Technol Assess. 2014;18(9):1-224.

60. Beusterien KM, Szabo SM, Kotapati S, et al. Societal preference values for advanced melanoma health states in the United Kingdom and Australia. Br J Cancer. 2009;101(3):387-89.

61. United States Bureau of Labor Statistics. Consumer Price Index, medical care. 2020. Accessed July 2020. https:// www.bls.gov/cpi/

62. Wolfe CM, Cognetta AB, Jr. Radiation therapy for nonmelanoma skin cancer, a cost comparison: 2016 coding changes to radiation therapy. J Am Acad Dermatol. 2017;77(3):e79-80.

63. National Institute for Health and Care Excellence. Single technology appraisal 439: vismodegib for treating basal cell carcinoma. November 22, 2017. Accessed November 2019. https://www.nice.org.uk/ guidance/ta489

64. National Comprehensive Cancer Network. Clinical Practice Guidelines in Oncology - Squamous Cell Skin Cancer (Version 3.2021). April 24, 2021. Accessed July 20, 2021. https://www.nccn.org/professionals/physician gls/pdf/squamous. pdf

65. Health Improvement Scotland, Scottish Intercollegiate Guidelines Network. Search filters. 2020. Accessed November 9, 2020. https://www.sign. ac.uk/what-we-do/methodology/ search-filters/

66. BMJ Best Practice. Study design search filters. Accessed July 16, 2021. https://bestpractice.bmj. com/info/toolkit/learn-ebm/ study-design-search-filters/

67. Lansbury L, Bath-Hextall F, Perkins W, Stanton W, Leonardi-Bee J. Interventions for non-metastatic squamous cell carcinoma of the skin: systematic review and pooled analysis of observational studies. BMJ. 2013;347:f6153. 
68. Lansbury L, Leonardi-Bee J, Perkins W, Goodacre T, Tweed JA, Bath-Hextall FJ. Interventions for nonmetastatic squamous cell carcinoma of the skin. Cochrane Database Syst Rev. 2010(4):Cd007869.

69. Trodello C, Pepper JP, Wong M, Wysong A. Cisplatin and cetuximab treatment for metastatic cutaneous squamous cell carcinoma: a systematic review. Dermatol Surg. 2017;43(1):40-49.

70. Shao XH, Xu YS, Zhang XQ, Li WF. Evidence based analysis of Cisplatin for treating patients with cutaneous squamous cell carcinoma. Asian Pac J Cancer Prev. 2014;15(22):9813-15.
71. Moher D, Liberati A, Tetzlaff J, Altman DG, Group P. Preferred reporting items for systematic reviews and metaanalyses: the PRISMA statement. J Clin Epidemiol. 2009;62(10):1006-12.

72. Higgins JP, Altman DG, Gøtzsche PC et al. The Cochrane Collaboration's tool for assessing risk of bias in randomised trials. BMJ. 2011;343:d5928.

73. National Institute for Health and Care Excellence. Methods for the development of NICE public health guidance (third edition). September 26, 2012. Accessed November 9, 2020. https://www.nice.org. uk/process/pmg 4
74. Wells GS, O'Connell D, Peterson J, Welch V, Losos M. The Newcastle-Ottawa Scale (NOS) for assessing the quality of nonrandomised studies in meta-analyses. 2021. Accessed June 20, 2021. http://www. ohri.ca/programs/clinical_epidemiology/ oxford.asp 